\title{
Improved red-response in thin film a-Si:H solar cells with soft-imprinted plasmonic back reflectors
}

\author{
Vivian E. Ferry, ${ }^{1, a)}$ Marc A. Verschuuren, ${ }^{2}$ Hongbo B. T. Li, ${ }^{3}$ Ruud E. I. Schropp, ${ }^{3}$ \\ Harry A. Atwater, ${ }^{1}$ and Albert Polman ${ }^{4}$ \\ ${ }^{1}$ Thomas J. Watson Laboratories of Applied Physics, California Institute of Technology, Pasadena, \\ California 91125, USA \\ ${ }^{2}$ Philips Research Laboratories, High Tech Campus 4, 5656 AE Eindhoven, The Netherlands \\ ${ }^{3}$ Utrecht University, Debye Institute for Nanomaterials Science, Section Nanophotonics, P.O. Box 80,000, \\ 3508 TA Utrecht, The Netherlands \\ ${ }^{4}$ Center for Nanophotonics, FOM-Institute AMOLF, Science Park 104, 1098XG Amsterdam, \\ The Netherlands
}

(Received 4 September 2009; accepted 6 October 2009; published online 3 November 2009)

\begin{abstract}
The impact of controlled nanopatterning on the Ag back contact of an n-i-p a-Si:H solar cell was investigated experimentally and through electromagnetic simulation. Compared to a similar reference cell with a flat back contact, we demonstrate an efficiency increase from $4.5 \%$ to $6.2 \%$, with a $26 \%$ increase in short circuit current density. Spectral response measurements show the majority of the improvement between 600 and $800 \mathrm{~nm}$, with no reduction in photocurrent at wavelengths shorter than $600 \mathrm{~nm}$. Optimization of the pattern aspect ratio using electromagnetic simulation predicts absorption enhancements over $50 \%$ at $660 \mathrm{~nm}$. (C) 2009 American Institute of Physics. [doi:10.1063/1.3256187]
\end{abstract}

Effective light trapping is a critical component of solar cell development. In typical thin film cells the thickness of the absorbing layer is governed by a tradeoff: the absorber must be optically thick to absorb a significant fraction of the incident photons but must also be of sufficient quality to enable minority carrier collection lengths larger than the material thickness. These dual requirements largely define current cost per Watt of photovoltaic power. Thin film Si solar cells using hydrogenated amorphous $\mathrm{Si}(\mathrm{a}-\mathrm{Si}: \mathrm{H})$ and nanocrystalline $\mathrm{Si}(\mathrm{nc}-\mathrm{Si}: \mathrm{H})$ are among the most well-developed thin film photovoltaic materials, but suffer from low diffusion lengths, and much work has been devoted to the optimization of surface texturing for light management. These surfaces are typically textured metal-oxide films, including $\mathrm{SnO}_{2}{ }^{1}$ in superstrate cells and sputtered films of $\mathrm{Ag}$ or $\mathrm{Al}$ and $\mathrm{ZnO}: \mathrm{Al}^{2,3}$ in substrate cells, where optimization of rms roughness has been explored but the topography is otherwise random. Surface texturing has also been achieved by incorporating roughness in electrically passive materials, such as plastic substrates ${ }^{4}$ and glass superstrates. ${ }^{5}$

Recently plasmonic nanostructures have garnered attention as a method for enhancing absorption in thin film photovoltaic absorber layers. ${ }^{6-9}$ While the introduction of additional metal structures can increase the Ohmic losses in the cell, through appropriate tailoring of the size, shape, and position of the nanostructures these losses can be minimized to enable overall spectral enhancement of photovoltaic performance. Compared to rough substrates, designed patterns allow for improved light management and controlled coupling of incident free space radiation to propagating guided wave modes. ${ }^{10,11}$ There are nonoptical device benefits to optimization of the improved light management as well: reducing the surface topography relative to a randomly structured rear

${ }^{\text {a)} E l e c t r o n i c ~ m a i l: ~ v i v i a n f @ c a l t e c h . e d u . ~}$ contact improves semiconductor film conformity and thereby electronic quality. ${ }^{12}$

A significant challenge is the fabrication of large-area patterns with precisely controlled nanoscale dimensions. In previous reported work, researchers have employed methods with varying degrees of size and position ordering, including island annealing, ${ }^{7}$ colloidal metal particles, ${ }^{8}$ and porous anodized aluminum oxide templates. ${ }^{13,14}$ Here we utilize nanoimprint lithography to pattern the back contact of an n-i-p a-Si:H solar cell and report on the enhancements in solar cell response relative to a planar reference cell using current density-voltage (J-V) and spectral response measurements. We also use full wave finite-difference time-domain (FDTD) simulations to model the spectral response and optimize the shape of the nanopatterned back reflector. We focus mainly on the red part of the spectrum, $600-800 \mathrm{~nm}$, where a-Si:H is weakly absorbing and the effect of light trapping is most pronounced.

Large-area nanopatterns were replicated using substrate conformal imprint lithography, a method that offers the advantages of inexpensive soft poly(dimethyl)siloxane (PDMS) stamps and delivers sub-50 $\mathrm{nm}$ resolution with wafer-scale pattern fidelity. ${ }^{15}$ The master pattern is a silicon wafer with 350 diameter holes, $200 \mathrm{~nm}$ deep, on a square lattice of pitch $513 \mathrm{~nm}$, made using laser interference lithography.

The surface of the master wafer is first modified with a fluorosilane antiadhesion monolayer. Next a 50 micron thick layer of high modulus PDMS layer is spin coated over the master and precured, then bonded onto a 200 micron thick glass sheet using low modulus PDMS ${ }^{16}$ and released from the master.

The pattern from the stamp is then transferred to the substrate. A $150 \mathrm{~nm}$ thick sol-gel based resist is applied by spin-coating over a $150 \mathrm{~mm}$ silicon wafer. The wafer is placed parallel to the stamp, at 100 micron distance. The stamp is attached by vacuum to a plate with grooves, which are sequentially pressurized to 20 mbar to contact the resist- 


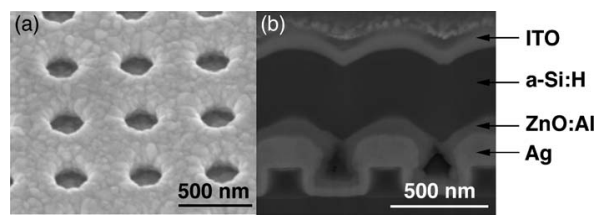

FIG. 1. (a) SEM image of a nanoimprinted pattern of holes after overcoating with Ag: The coated holes are $225 \mathrm{~nm}$ in diameter, $240 \mathrm{~nm}$ deep, and have a pitch of $513 \mathrm{~nm}$. (b) SEM image showing a FIB cross section of a fully fabricated n-i-p a-Si:H solar cell grown on the patterned back contact.

coated wafer. The sol-gel reacts at room temperature in 10 min to form a solid. The grooves are then sequentially evacuated, which results in a smooth release of the stamp from the imprinted patterns. The stamps can be used for over 2000 sol-gel imprints without observed pattern degradation. The replicated sol-gel hole array is 88 wt \% silicon oxide, nonabsorbing, and stable in air up to $450{ }^{\circ} \mathrm{C}$.

To form the back contact, the patterned sol-gel layer is sputter-coated with $200 \mathrm{~nm} \operatorname{Ag}(1 \% \mathrm{Pd})$, as illustrated in the scanning electron micrograph (SEM) image in Fig. 1(a). The metal holes are $225 \mathrm{~nm}$ in diameter after coating. The back contact for a flat reference cell was made by evaporating 200 $\mathrm{nm}$ of Ag on glass. Both cells were then processed side-byside in the remaining steps to ensure identical deposition conditions. A $100 \mathrm{~nm} \mathrm{ZnO:Al} \mathrm{spacer} \mathrm{layer} \mathrm{was} \mathrm{sputtered} \mathrm{on}$ top of the Ag contact, followed by standard n-i-p a-Si:H cell deposition using $13.56 \mathrm{MHz}$ plasma-enhanced chemical vapor deposition with an intrinsic layer thickness of $500 \mathrm{~nm} .{ }^{17}$ An $80 \mathrm{~nm}$ indium tin oxide (ITO) contact was sputtered on top, which also serves as an antireflection coating. Finally a metal grid was evaporated over the ITO using a contact mask. The active area of the cell is $0.13 \mathrm{~cm}^{2}$. Figure $1(\mathrm{~b})$ shows a cross section of a cell after fabrication on top of the patterned cell, made using focused ion beam (FIB) milling. The different layers can be clearly identified and the holes are conformally coated with $\mathrm{Ag}$ and $\mathrm{ZnO}: \mathrm{Al}$.

Figure 2 illustrates the current-voltage characteristics of the flat and patterned n-i-p a-Si:H cells, for the best cell of each type. The $\mathrm{J}-\mathrm{V}$ characteristics were measured with a solar simulator under one sun illumination (AM1.5G, $100 \mathrm{~mW} / \mathrm{cm}^{2}$ ). The patterned cell exhibits a $26 \%$ higher short circuit current density $\left(\mathrm{J}_{\mathrm{sc}}\right)$ than the flat cell, demonstrating an increased optical path length in the device. The open circuit voltage $\left(\mathrm{V}_{\mathrm{oc}}\right)$ shows a slight decrease, by $2 \%$. Combined, there is a significant increase in efficiency from $4.5 \%$ to $6.2 \%$ due to the patterned metal back contact. Sev-

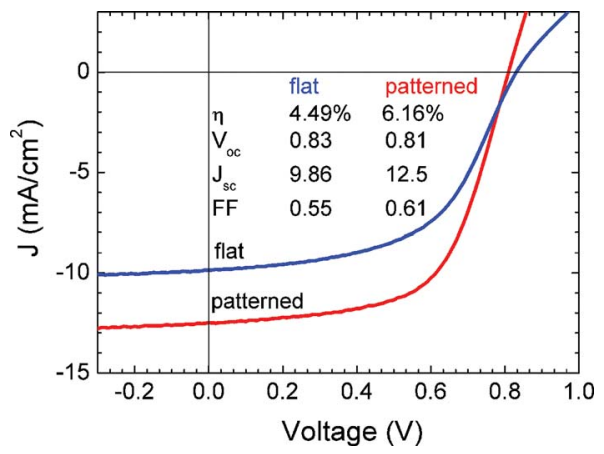

FIG. 2. (Color online) Best J-V measurements of the flat reference and patterned n-i-p a-Si:H cells. Inset shows the cell characteristics for each device.

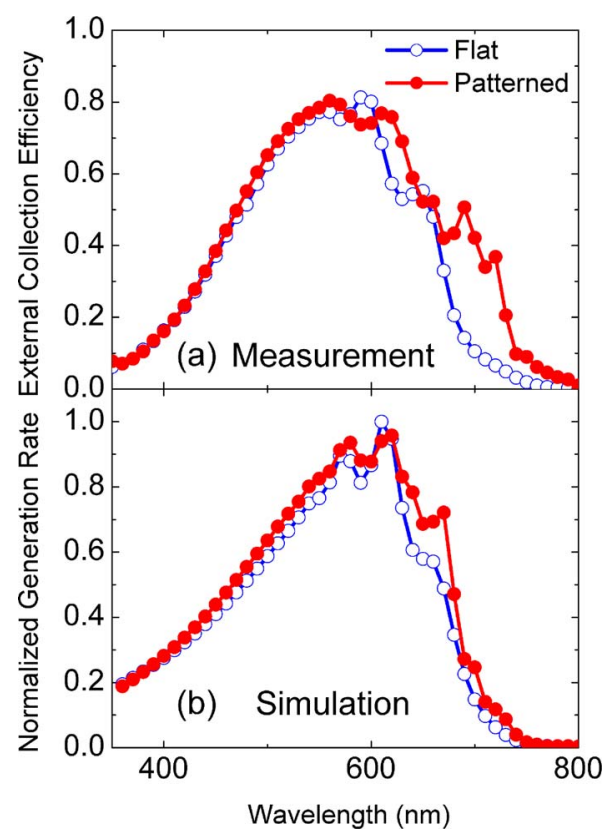

FIG. 3. (Color online) (a) Measured spectral response curves for the flat and patterned cells under bias voltage $=-1$ V. (b) Simulated normalized electron generation rates for flat and patterned cells.

eral cells of each type were measured, with a variance in the absolute efficiency of 0.1 for the reference cell and 0.13 for the patterned cell. The variation in the fill factor is attributed to differences between the electrical contacts. It is important to note that the patterned cell does not suffer from a reduced series resistance or fill factor.

To better understand the nature of the enhancement, we measured spectral response curves for the two sets of devices. Figure 3(a) shows the spectral response measured under a reverse bias of $-1 \mathrm{~V}$, which facilitates comparison to optical simulation results by sweeping out all of the generated electrons. At wavelengths shorter than $550 \mathrm{~nm}$ there is little difference between the flat and patterned cells. This contrasts with designs for light trapping incorporating metal nanoparticles on top, which often see a decrease in efficiency in this part of the spectrum. ${ }^{7,9}$ At these wavelengths most of the light is absorbed in the $500 \mathrm{~nm}$ thick i-a-Si:H layer before interacting with the scattering layer. At wavelengths longer than $600 \mathrm{~nm}$ there is a significant difference in photocurrent between the two cells. Integrating over the 600 $800 \mathrm{~nm}$ region a $51 \%$ increase in photocurrent is found.

We also use electrodynamic modeling to understand the optical absorption and optimize the dimensions of the nanostructured back reflector design. Simulations are done using three-dimensional full field FDTD simulation, with a rendered $\mathrm{Si} / \mathrm{ZnO}: \mathrm{Al} / \mathrm{Ag}$ structure that is an approximation to the experimental back reflector geometry as found in SEM [Fig. 3(b)]. The thin $\mathrm{n}$ and $\mathrm{p}$ layers (approximately $20 \mathrm{~nm}$ ) were neglected, with the entire $500 \mathrm{~nm}$ a-Si:H layer taken as intrinsic and optically active. The complex refractive index data for the a-Si:H layer was based on previously measured optical constants. ${ }^{17}$ The refractive index data for the ITO and $\mathrm{ZnO}$ :Al layers were $2.08+0.004 i$ and $1.93+0.004 i$, respectively, with slight dispersion as measured using ellipsometry. Data for Ag were taken using a Lorentz-Drude model fitted to data from Palik. ${ }^{18}$ As a simple topological approximation to the experimentally observed patterned structure, the inte- 


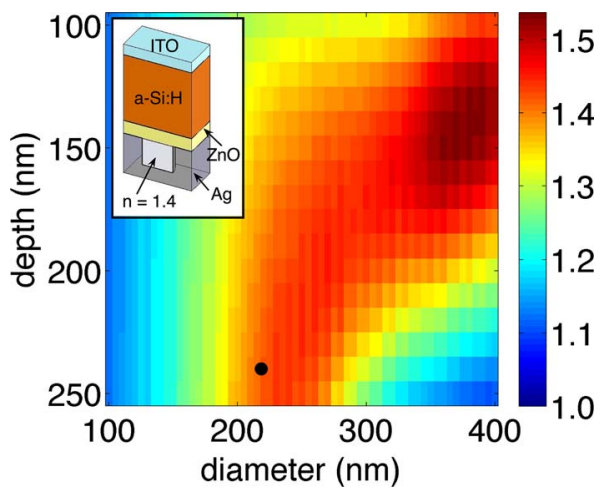

FIG. 4. (Color online) Calculated absorption enhancement for different $\mathrm{Ag}$ hole diameters and depths at $\lambda=660 \mathrm{~nm}$. The solid dot represents the experimental configuration. Inset: Geometry of the simulation cell.

rior of the hole was modeled with an effective index of $\mathrm{n}$ $=1.4$, between that of $\mathrm{ZnO}$ and vacuum, as illustrated in the inset of Fig. 4. The normalized optical generation rate $G_{\text {opt }}$ was calculated at each wavelength from the divergence of the Poynting vector according to $G_{\text {opt }}=\frac{1}{2 \hbar \omega} \Re[\nabla \cdot \mathbf{S}]=\frac{\epsilon^{\prime}|\mathbf{E}|^{2}}{2 \hbar}$, where $\epsilon^{\prime \prime}$ is the imaginary part of the complex permittivity and $\mathbf{E}$ is the electric field and $G_{o p t}$ was integrated over the simulation volume containing a-Si:H. ${ }^{19,20}$

Figure 3(b) illustrates the calculated normalized generation rate over the range of the solar spectrum where a-Si:H is absorbing. As this is exclusively an optical model and does not account for the efficiency of carrier collection, the results are most comparable to a spectral response measurement under reverse bias. The overall spectral shape for both the flat and patterned cells corresponds well to the measured spectral response data, reproducing the predominant spectral peaks and shoulders, indicating that the optical absorption model is reasonable for comparison to the experimental data. A clear enhanced red-response is calculated for the patterned cell, though slightly less pronounced than in the experiment.

Given that the model correctly predicts the experimental results, it was used to search for an optimized scattering pattern design. Figure 4 shows the calculated absorption enhancement due to light trapping for a range of hole diameters and depths at $\lambda=660 \mathrm{~nm}$. The experimental configuration, which is shown by the solid dot, has an enhancement of $42 \%$ compared to the control sample. Increasing the diameter of the hole further to $370 \mathrm{~nm}$ and reducing the depth to $140 \mathrm{~nm}$ will increase the absorption enhancement in the a-Si:H to $54 \%$.

In conclusion, we have shown that including periodic nanostructures on the back contact of an n-i-p a-Si:H solar cell enhances the red-response of the device, predominantly through a $26 \%$ increase in $\mathrm{J}_{\mathrm{sc}}$. The overall cell efficiency improves from $4.5 \%$ to $6.2 \%$ due to the patterns. We observe that the photocurrent enhancements are largest at wavelengths longer than $600 \mathrm{~nm}$; no decrease in performance is found at shorter wavelengths. The nanopatterns were made using an inexpensive and scalable process that allows for exact control of the feature size, shape, and arrangement. Optical electromagnetic modeling compares well to the experimental data, and predicts further optimization of the pattern is possible. This combination of these features makes this a model system for investigating the achievable photovoltaic efficiency improvements due to controlled light management.

We gratefully acknowledge $\mathrm{H}$. Zeijlemaker for FIB assistance and C.H.M. van der Werf for solar cell deposition. The Caltech portion of this work was supported by the Department of Energy under Contract Number DE-FG0207ER46405. Work at AMOLF is part of the research program of FOM which is financially supported by NWO. This work is part of the Global Climate and Energy Project (GCEP). This work is also a part of NANONED, a technology program of the Dutch Ministry of Economic Affairs.

${ }^{1}$ K. Sato, Y. Gotoh, Y. Wakayama, Y. Hayashi, K. Adachi, and H. Nishimura, Rep. Res. Lab. Asahi Glass Co. Ltd. 42, 129 (1992).

${ }^{2}$ R. H. Franken, R. L. Stolk, H. Li, C. H. M. van der Werf, J. K. Rath, and R. E. I. Schropp, J. Appl. Phys. 102, 014503 (2007).

${ }^{3}$ J. Müller, B. Rech, J. Springer, and M. Vanecek, Sol. Energy 77, 917 (2004).

${ }^{4}$ F.-J. Haug, T. Söderström, O. Cubero, V. Terrazzoni-Daudrix, and C. Ballif, J. Appl. Phys. 104, 064509 (2008).

${ }^{5}$ K. Niira, H. Senta, H. Hakuma, M. Komoda, H. Okui, K. Fukui, H. Arimune, and K. Shirasawa, Sol. Energy Mater. Sol. Cells 74, 247 (2002).

${ }^{6}$ V. E. Ferry, L. A. Sweatlock, D. Pacifici, and H. A. Atwater, Nano Lett. 8, 4391 (2008)

${ }^{7}$ F. J. Beck, A. Polman, and K. R. Catchpole, J. Appl. Phys. 105, 114310 (2009).

${ }^{8}$ D. Derkacs, S. H. Lim, P. Matheu, W. Mar, and E. T. Yu, Appl. Phys. Lett. 89, 093103 (2006).

${ }^{9}$ K. R. Catchpole and A. Polman, Appl. Phys. Lett. 93, 191113 (2008).

${ }^{10}$ C. Eisele, C. E. Nebel, and M. Stutzmann, J. Appl. Phys. 89, 7722 (2001).

${ }^{11}$ C. Haase and H. Stiebig, Appl. Phys. Lett. 91, 061116 (2007).

${ }^{12}$ R. E. I. Schropp, J. K. Rath, and H. Li, J. Cryst. Growth 311, 760 (2009).

${ }^{13}$ K. Nakayama, K. Tanabe, and H. A. Atwater, Appl. Phys. Lett. 93, 121904 (2008).

${ }^{14}$ H. Sai and M. Kondo, J. Appl. Phys. 105, 094511 (2009).

${ }^{15}$ M. Verschuuren and H. van Sprang, Mater. Res. Soc. Symp. Proc. 1002, N03 (2007).

${ }^{16}$ T. W. Odom, J. C. Love, D. B. Wolfe, K. E. Paul, and G. M. Whitesides, Langmuir 18, 5314 (2002).

${ }^{17}$ R. E. I. Schropp and M. Zeman, Amorphous and Microcrystalline Silicon Solar Cells: Modeling, Materials, and Device Technology (Kluwer Academic, Boston, 1998).

${ }^{18}$ A. D. Rakic, A. B. Djurisic, J. M. Elazar, and M. L. Majewski, Appl. Opt. 37, 5271 (1998).

${ }^{19}$ M. D. Kelzenberg, M. C. Putnam, D. B. Turner-Evans, N. S. Lewis, and H. A. Atwater, Proceedings of the 34th IEEE Photovoltaics Specialists Conference, June 7-12, 2009, Philadelphia, (unpublished).

${ }^{20}$ A. D. Yaghjian, IEEE Trans. Antennas Propag. 55, 1495 (2007). 